\title{
Effect of FOLFOX4 combined with Brucea javanica emulsion on VEGF in patients with gastric cancer
}

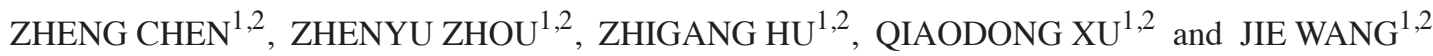 \\ ${ }^{1}$ Department of General Surgery, Guangdong Province Key Laboratory of Malignant Tumor Epigenetics and \\ Gene Regulation, Research Center for Medicine; ${ }^{2}$ Department of General Surgery, Sun Yat-sen Memorial Hospital, \\ Sun Yat-sen University, Haizhu, Guangzhou 510220, P.R. China
}

Received July 17, 2017; Accepted October 27, 2017

DOI: $10.3892 / \mathrm{ol} .2017 .7429$

\begin{abstract}
The aim of the study was to investigate the effect of FOLFOX4 regimen combined with Brucea javanica emulsion on the content of serum vascular endothelial growth factor (VEGF) in patients with gastric cancer, and to evaluate the efficacy of FOLFOX4 regimen combined with Brucea javanica emulsion on gastric cancer. A total of 60 patients with gastric ulcer were selected as the normal group, and another 150 patients with gastric cancer were randomly divided into two groups, of which 75 patients with gastric cancer treated with FOLFOX4 regimen after operation were selected as the control group and another 75 patients with gastric cancer treated with FOLFOX4 regimen combined with Brucea javanica emulsion after operation were selected as the experimental group. The serum VEGF levels of patients in the different groups before operation, after chemotherapy for 3 times and at 1 and 3 months after chemotherapy were compared via enzyme-linked immunosorbent assay. The content of serum VEGF in patients with gastric cancer before operation was significantly higher than that in the normal group $(\mathrm{P}<0.05)$. After administration of chemotherapy 3 times, the content of serum VEGF in the control group had no significant difference from that in the experimental group $(\mathrm{P}>0.05)$. Additionally, at 1 and 3 months after chemotherapy, the content of serum VEGF of patients in the experimental group were significantly lower than those in the control group $(\mathrm{P}<0.05)$. The results showed that FOLFOX4 regimen combined with Brucea javanica emulsion can significantly reduce the level of serum VEGF in patients with gastric cancer, and has a certain effect
\end{abstract}

Correspondence to: Dr Jie Wang, Guangdong Province Key Laboratory of Malignant Tumor Epigenetics and Gene Regulation, Research Center for Medicine; Department of General Surgery, Sun Yat-sen Memorial Hospital, Sun Yat-sen University, 33 Yingfeng Road, Haizhu, Guangzhou 510220, P.R. China

E-mail: jzw3qs@163.com

Key words: chemotherapy, Brucea javanica emulsion, gastric cancer, vascular endothelial growth factor in reducing the postoperative recurrence rate of gastric cancer and improving the effect of chemotherapy.

\section{Introduction}

Gastric cancer is one of the most common malignant tumors and occurs frequently in China with approximately 400,000 new cases each year, accounting for $42 \%$ of new cases of gastric cancer worldwide (1). In addition, its incidence shows an increasing trend year by year. Gastric cancer has a high metastatic and recurrence rate, poor prognosis and low 5-year survival rate (2). Thus, investigations on reducing the postoperative metastatic and recurrence rate has important clinical significance.

FOLFOX 4 is the first-line regimen of postoperative chemotherapy for gastric cancer, but it has great toxic side effects, such as hematologic toxicity and peripheral neurotoxicity (3). Consequently, how to modify FOLFOX4 regimen, improvement of the chemotherapeutic efficacy, reduction of toxic and side effects and the metastatic and recurrence rate of gastric cancer, as well as the prevention of drug resistance reversal are clinical issues that remain to be resolved. Brucea javanica oil is the fatty oil extracted from the dried and mature fruit of Brucea javanica, a kind of quassia plant. Clinical studies have confirmed that it has a good therapeutic effect on a variety of tumors, such as lung, liver, breast cancer and colorectal cancer (4), and it can improve chemotherapy efficacy and reduce the adverse reaction of chemotherapeutic drugs when combined with them (5). Brucea javanica exerts antitumor pharmacological effects through the inhibition of tumor cell DNA production, tumor cell proliferation and activity and drug resistance reversal of DNA topoisomerase II (6).

In this study, the effects of FOLFOX4 regimen combined with Brucea javanica emulsion and simple FOLFOX4 regimen on serum VEGF content and postoperative recurrence rate of patients with gastric cancer were compared, to provide a clinical basis for improving the FOLFOX4 regimen for postoperative chemotherapy of gastric cancer.

\section{Materials and methods}

General materials. Sixty gastric ulcer patients treated from January 2013 to January 2014 in the Sun Yat-sen Memorial Hospital were selected as the normal group. Of the 60 patients, 
Table I. Comparisons of VEGF content in different patients between groups (pg/ml).

\begin{tabular}{|c|c|c|c|c|}
\hline Variables & Before operation & $\begin{array}{l}\text { After chemotherapy } \\
\text { for } 3 \text { times }\end{array}$ & $\begin{array}{l}\text { At } 1 \text { month } \\
\text { after chemotherapy }\end{array}$ & $\begin{array}{l}\text { At } 3 \text { months } \\
\text { after chemotherapy }\end{array}$ \\
\hline Normal group & $91.72 \pm 14.36$ & $89.32 \pm 10.69$ & $85.24 \pm 24.73$ & $82.91 \pm 31.28$ \\
\hline Control group & $392.4 \pm 48.19$ & $359.7 \pm 39.42$ & $289.3 \pm 41.65$ & $301.9 \pm 26.32$ \\
\hline P-value & $<0.05$ & $<0.05$ & $<0.05$ & $<0.05$ \\
\hline Normal group & $91.72 \pm 14.36$ & $89.32 \pm 10.69$ & $85.24 \pm 24.73$ & $82.91 \pm 31.28$ \\
\hline Experimental group & $405.8 \pm 37.62$ & $353.5 \pm 47.39$ & $215.2 \pm 24.68$ & $159.6 \pm 23.71$ \\
\hline P-value & $<0.05$ & $<0.05$ & $<0.05$ & $<0.05$ \\
\hline Control group & $392.4 \pm 48.19$ & $359.7 \pm 39.42$ & $289.3 \pm 41.65$ & $301.9 \pm 26.32$ \\
\hline Experimental group & $405.8 \pm 37.62$ & $353.5 \pm 47.39$ & $215.2 \pm 24.68$ & $159.6 \pm 23.71$ \\
\hline P-value & $>0.05$ & $>0.05$ & $<0.05$ & $<0.05$ \\
\hline
\end{tabular}

41 were males and 19 were females, with an average age of 49 years. A total of 150 patients with gastric cancer confirmed via pathological examination were randomly divided into the control and experimental groups. Of the 150 patients there were 75 patients in the control group, including 42 males and 33 females with an average age of 52 years, and there were 75 patients in the experimental group, including 40 males and 35 females with an average age of 55 years. The results of blood routine, liver and kidney function and electrocardiogram for all the patients before treatment were normal, and there was no obvious metastasis in liver, spleen and pancreas in upper abdominal ultrasonography and $\mathrm{CT}$ examination. According to the statistical analysis, there were no significant differences in the general materials of all the subjects, and they were comparable.

Patients in the control and experimental groups provided written informed consent regarding surgery and chemotherapy. Approval for the study was obtained from the Ethics Committee of Sun Yat-sen Memorial Hospital.

Treatment methods. Patients in the normal group were treated with regular treatment of ulcer, while patients in the control group were treated with FOLFOX4 chemotherapy. On day 1 the patients were treated with intravenous drip of $85 \mathrm{mg} / \mathrm{m}^{2}$ oxaliplatin $+500 \mathrm{ml} 5 \%$ glucose solution once time based on the surface area formula, and then intravenous drip of $200 \mathrm{mg} / \mathrm{m}^{2}$ leucovorin $+100 \mathrm{ml}$ sodium chloride solution was applied, followed by small amount of 5-fluorouracil via intravenous drip, and intravenous drip of $600 \mathrm{mg} / \mathrm{m}^{2}$ 5-fluorouracil $+250 \mathrm{ml} 5 \%$ glucose solution. The method used on day 2 was the same as that in day 1 after the intravenous drip in day 1 was finished, and the same dosage of leucovorin and 5-fluorouracil was given to the patients. Subsequently, the administration methods in day 1 and 2 were repeated with an interval of 14 days: A total of 16 days as 1 cycle and 6 cycles as 1 course of treatment. The chemotherapy treatment of the experimental group was the same as that of the control group, but $30 \mathrm{ml}$ Brucea javanica injection $+250 \mathrm{ml}$ sodium chloride solution were given via intravenous drip between the administrations of leucovorin and 5-fluorouracil. The treatment cycle was the same as that of the control group.
Main experimental equipment and reagents. Equipment used included the Eppendorf pipetter (Eppendorf, Hamburg, Germany), BIO-RAD550 microplate reader (Bio-Rad Laboratories, Inc., Hercules, CA, USA) and human VEGF ELISA kit (HCB, Ontario, Canada).

Specimen collection. Fasting peripheral venous blood $(5 \mathrm{ml})$ was collected from all the subjects before treatment, after chemotherapy for 3 times and at 1 and 3 months after chemotherapy, followed by centrifugation at $3,000 \mathrm{x} \mathrm{g}$ for $10 \mathrm{~min}$ to separate the serums. Then the supernatant was placed in the refrigerator at $-20^{\circ} \mathrm{C}$ for later inspection.

Statistical analysis. SPSS 16.0 (SPSS, Inc., Chicago, IL, USA) software was used for the statistical analysis. The measurement data were presented as mean \pm standard deviation, and the t-test was used for the pairwise comparison of sample means and Chi-square test was used for the enumeration data. $\mathrm{P}<0.05$ was considered to indicate a statistically significant difference.

\section{Results}

Comparisons of VEGF content at different time points. The serum VEGF contents of different patients for the groups at 4 different time points are shown in Table I. The results showed that the VEGF content of patients in the control or experimental group was significantly higher than that in the normal group, and the differences were statistically significant $(\mathrm{P}<0.05)$. There were no significant differences between the experimental and control groups before treatment and after chemotherapy for 3 times. At 1 month after chemotherapy, the VEGF content of patients in experimental group was significantly lower than that in control group, and the difference was statistically significant $(\mathrm{P}<0.05)$. In addition, at 3 months after chemotherapy, the VEGF content of patients in the experimental group was significantly lower than that in the control group, and the difference was statistically significant $(\mathrm{P}<0.05)$.

Serum VEGF contents for the three groups at different time points. The serum VEGF contents of different patients among groups at 4 different time points are shown in Table II. The 
Table II. Comparisons of VEGF content in different patients within the group $(\mathrm{pg} / \mathrm{ml})$.

\begin{tabular}{lccc}
\hline & \multicolumn{2}{c}{ Groups } & Experimental \\
\cline { 2 - 4 } Variables & Normal & Control & $405.8 \pm 37.62$ \\
Before operation & $91.72 \pm 14.36$ & $392.4 \pm 48.19$ & $353.5 \pm 47.39$ \\
After chemotherapy for 3 times & $89.32 \pm 10.69$ & $359.7 \pm 39.42$ & $>0.05$ \\
P-value & $>0.05$ & $>0.05$ & $353.5 \pm 47.39$ \\
After chemotherapy for 3 times & $89.32 \pm 10.69$ & $359.7 \pm 39.42$ & $215.2 \pm 24.68$ \\
At 1 month after chemotherapy & $85.24 \pm 24.73$ & $289.3 \pm 41.65$ & $<0.05$ \\
P-value & $>0.05$ & $<0.05$ & $215.2 \pm 24.68$ \\
At 1 month after chemotherapy & $85.24 \pm 24.73$ & $301.9 \pm 26.32$ & $159.6 \pm 23.71$ \\
At 3 months after chemotherapy & $82.91 \pm 31.28$ & $>0.05$ & $<0.05$ \\
P-value & $>0.05$ & & \\
\hline
\end{tabular}

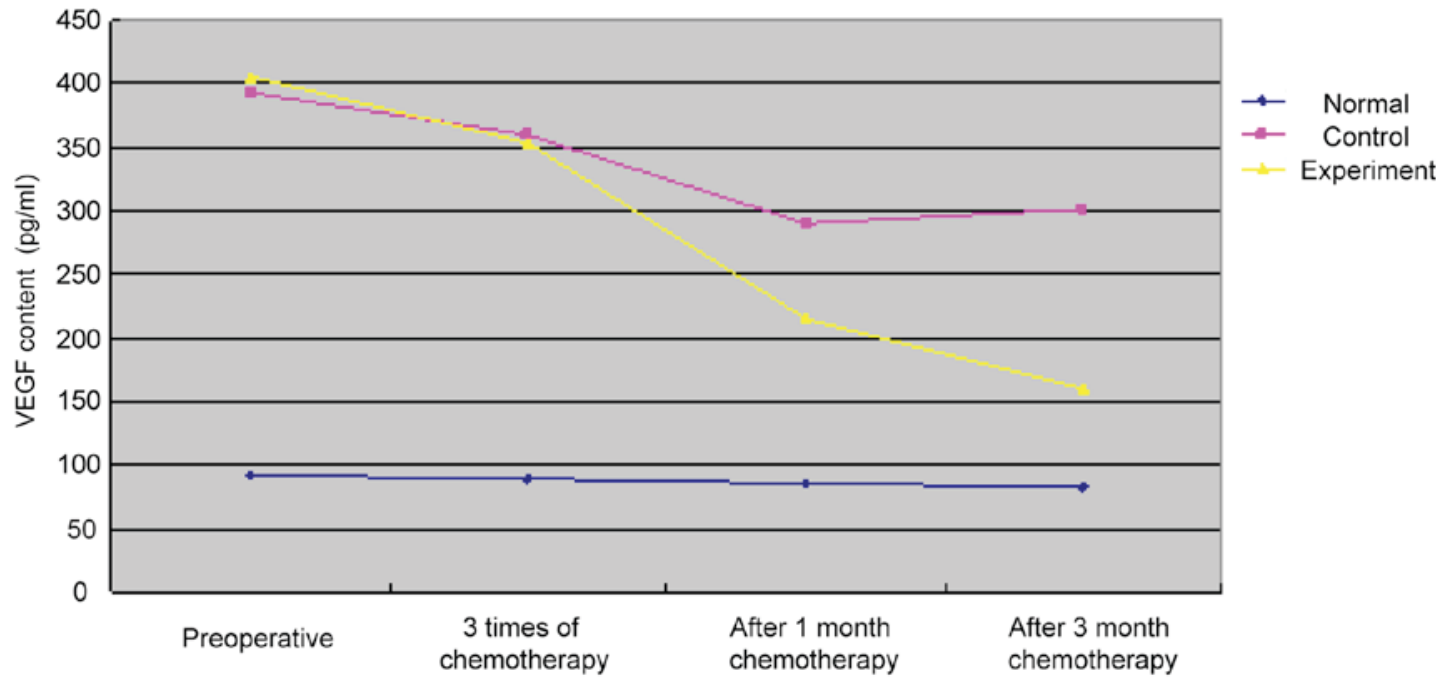

Figure 1. Tendency chart of VEGF content of patients in the three groups at different time points (pg/ml). VEGF, vascular endothelial growth factor.

results showed that there were no significant differences among the three groups $(\mathrm{P}>0.05)$ in the VEGF contents before operation and after chemotherapy for 3 times. An increase in chemotherapy, resulted in VEGF contents in the control and experimental groups being decreased and the differences were statistically significant $(\mathrm{P}<0.05)$ after chemotherapy for 3 times and at 1 month after chemotherapy. The VEGF contents of patients in the control group had no significant decrease at 1 and 3 months after chemotherapy, but those in the normal and experimental groups were obviously decreased, and the differences were statistically significant $(\mathrm{P}<0.05)$.

Tendency of VEGF contents in the three groups at different time points. The VEGF contents of patients in the three groups at different time points are shown in Fig. 1. The results showed that the VEGF content of patients in the control group was gradually decreased with the advance of chemotherapy, but slightly increased at 3 months after chemotherapy. In addition, the VEGF content of patients in the experimental group was also decreased gradually with the increase of chemotherapy, and the decreasing rate was higher than that in the control group at 1 month after chemotherapy.

\section{Discussion}

Gastric cancer is the most common malignant tumor globally, and its morbidity and mortality rates rank second worlwide (7). The incidence of gastric cancer in different countries, ethnicities, genders and ages is not the same. The number of male patients with gastric cancer is more than that with female patients (about 2:1). Most patients with gastric cancer are middle-aged and elderly individuals aged 50-70 years, showing a young tendency with the changes in lifestyle and diet (8). Owning to its high incidence, metastatic and recurrence rates, gastric cancer has become a serious health problem in China. The occurrence and development of gastric cancer is a multifactor and multi-step process. The proliferation and apoptosis of gastric mucosal epithelial cells under the physiological condition is a dynamic equilibrium process, which is controlled by a variety of oncogenes, tumor suppressor genes and growth factors, such as epidermal growth factor and VEGF. VEGF is closely related to tumor growth, which can promote the growth of blood vessels, proliferation of vascular endothelial cells, and migration of vascular endothelial cells and change the permeability of blood 
vessels (9). If gastric epithelial cells proliferate excessively without apoptosis in pathological conditions, gastric disease may develop into gastric cancer (10). The present study, by comparing the VEGF levels of patients in the normal, control and experimental groups before treatment, found that the VEGF level in patients with gastric cancer was significantly higher than that in patients with gastric disease, and the difference was statistically significant $(\mathrm{P}<0.05)$, indicating that the development of gastric cancer is closely related to VEGF, and VEGF may be a target of gastric cancer treatment.

At present, the only possible radical method of gastric cancer is surgical resection and lymph node dissection around the lesions (11). The staging, infiltration degree and diffusion range of gastric cancer determine the effect of gastric cancer surgery. The survival rate can be improved after the surgical resection of partial stomach if there is no metastasis of gastric cancer found in examination. The purpose of chemotherapy is to reduce and remove the cancer foci and prevent its metastasis and recurrence (12). There are lots of common chemotherapy regimens for gastric cancer, one of which is FOLFOX4, including three kinds of drugs: oxaliplatin, leucovorin and 5-fluorouracil. Oxaliplatin is the third generation of platinum compound that plays the anticancer effect via blocking the DNA replication and transcription of cancer cells, and the curative effect is better than cisplatin without crossresistance (13). 5-Fluorouracil is the thymidylate synthase inhibitor, and belongs to the cell cycle-specific drug, which prevents cell division and proliferation via intervention in nucleic acid metabolism of cancer cells. Calcium folinate can be used as its sensitizer when combined with 5-fluorouracil. As with chemotherapeutic drugs, the FOLFOX4 regimen, a kind of pure chemical drug, has a certain cytotoxicity, hematologic toxicity and peripheral neurotoxicity, and may also lead to drug resistance reversal (14). However, the participation of Chinese traditional medicine can play a coordinating role to improve the efficacy of chemotherapy drugs, reduce the toxic and side effects and prevent the multi-drug resistance reversal and metastasis or recurrence of tumors. Brucea javanica oil is the fat oil extracted from the peel and seed of Brucea javanica via petroleum ether reflux, containing the oil and various fatty acids, among which the oleic acid and linoleic acid have strong anticancer activity because of the specific affinity with the cancer cell membrane (15). It can achieve the anticancer effect in a variety of ways, such as affecting the tumor cell cycle, inhibiting DNA synthesis, inducing tumor cell apoptosis, inhibiting cancer cell growth, improving immune function and reversing tumor multi-drug resistance (16).

Brucea javanica emulsion can be used for the treatment of a variety of cancers, such as colorectal, breast and lung cancer. However, there are few reports about the treatment of gastric cancer with Brucea javanica emulsion at present, and the way to achieve the treatment purpose is not clear, either. In the present study, the VEGF levels in the experimental and control groups after chemotherapy for 3 times were compared and it was found that there was no significant difference in VEGF content between the two groups. Additionally, the advance of chemotherapy, the comparisons of VEGF levels in patients between the experimental and control groups at 1 month and 3 months after chemotherapy showed that the levels in the experimental group were significantly lower than those in the control group. It suggested that Brucea javanica emulsion, as a kind of Chinese patent medicine, is slow to take effect without obvious advantages compared with the chemotherapeutic drug alone in the middle of chemotherapy. However, the chemotherapeutic effect on gastric cancer was increased with the passage of time after the Brucea javanica emulsion was combined, indicating that Brucea javanica may improve the curative effect of chemotherapy drugs by inhibiting the angiogenesis of gastric cancer and proliferation of gastric cancer cells, and promoting its apoptosis.

In summary, the FOLFOX4 regimen combined with Brucea javanica emulsion in the treatment of patients with gastric cancer after operation can significantly reduce the VEGF content compared with the single administration of FOLFOX4, and the difference is statistically significant $(\mathrm{P}<0.05)$, which indicates that Brucea javanica emulsion may inhibit the development of gastric cancer by inhibiting the angiogenesis of gastric cancer, and the drug combination is helpful to improve the curative effect of chemotherapy. Next, methyl thiazolyl tetrazolium assay and flow cytometry can be used to detect the apoptosis, to further study whether the antitumor effect of Brucea javanica emulsion is realized by inhibiting the apoptosis of gastric cancer cells. In conclusion, it was found in this study that the effect of chemotherapy on patients with gastric cancer can be improved by FOLFOX4 regimen combined with Brucea javanica emulsion, and it is worthy of clinical recommendation.

\section{References}

1. Patru CL, Surlin V, Georgescu I and Patru E: Current issues in gastric cancer epidemiology. Rev Med Chir Soc Med Nat Iasi 117: 199-204, 2013.

2. Schmidt T, Alldinger I, Blank S, Klose J, Springfeld C, Dreikhausen L, Weichert W, Grenacher L, Bruckner T, Lordick F, et al: Surgery in oesophago-gastric cancer with metastatic disease: Treatment, prognosis and preoperative patient selection. Eur J Surg Oncol 41: 1340-1347, 2005.

3. Jeon EK, Hong SH, Kim TH, Jung SE, Park JC, Won HS, Ko YH, Rho SY and Hong YS: Modified FOLFIRI as second-line chemotherapy after failure of modified FOLFOX-4 in advanced gastric cancer. Cancer Res Treat 43: 148-153, 2011.

4. Yan Z,Zhang B, Huang Y, Qiu H, Chen P and Guo GF: Involvement of autophagy inhibition in Brucea javanica oil emulsion-induced colon cancer cell death. Oncol Lett 9: 1425-1431, 2015.

5. Hu Y, Wan XJ, Pan LL, Zhang SH and Zheng FY: Effects of Brucea javanica oil emulsion on human papilloma virus type 16 infected cells and mechanisms research. Zhongguo Zhong Xi Yi Jie He Za Zhi 33: 1545-1551, 2013 (In Chinese).

6. Ohtsu A, Ajani JA, Bai YX, Bang YJ, Chung HC, Pan HM, Sahmoud T, Shen L, Yeh KH, Chin K, et al: Everolimus for previously treated advanced gastric cancer: Results of the randomized, double-blind, phase III GRANITE-1 study. J Clin Oncol 31: 3935-3943, 2013.

7. Yamamoto D, Hamada Y, Okazaki S, Kawakami K, Kanzaki S, Yamamoto C and Yamamoto M: Metastatic gastric tumor from renal cell carcinoma. Gastric Cancer 12: 170-173, 2009.

8. Chung HW, Lee SY, Han HS, Park HS, Yang JH, Lee HH and So Y: Gastric cancers with microsatellite instability exhibit high fluorodeoxyglucose uptake on positron emission tomography. Gastric Cancer 16: 185-192, 2013.

9. Wen J, Linghu EQ, Yang YS, Liu QS, Yang J and Lu ZS: Associated risk factor analysis for positive resection margins after endoscopic submucosal dissection in early-stage gastric cancer. J BUON 20: 421-427, 2015.

10. He YX, Song XH, Zhao ZY and Zhao H: HOXA13 upregulation in gastric cancer is associated with enhanced cancer cell invasion and epithelial-to-mesenchymal transition. Eur Rev Med Pharmacol Sci 21: 258-265, 2017. 
11. Ye M, Jin K, Xu G, Lin F, Zhou Q, Tao K and Tao F: Short-and long-term outcomes after conversion of laparoscopic total gastrectomy for gastric cancer: A single-center study. J BUON 22: 126-133, 2017.

12. Chung HW, Lee SY, Han HS, Park HS, Yang JH, Lee HH and So Y: Gastric cancers with microsatellite instability exhibit high fluorodeoxyglucose uptake on positron emission tomography. Gastric Cancer 16: 185-192, 2013.

13. Wen J, Linghu EQ, Yang YS, Liu QS, Yang J and Lu ZS: Associated risk factor analysis for positive resection margins after endoscopic submucosal dissection in early-stage gastric cancer. J BUON 20: 421-427, 2015.

14. He YX, Song XH, Zhao ZY and Zhao H: HOXA13 upregulation in gastric cancer is associated with enhanced cancer cell invasion and epithelial-to-mesenchymal transition. Eur Rev Med Pharmacol Sci 21: 258-265, 2017.
15. Wu YC, Zhang YC, Dai GX, Wang LJ and Jin-Kea YE: Clinical observation of Bruceolic oil emulsion combined with FOLFOX4 in treating advanced carcinoma of stomach. Med Pharm J Chin Peoples Liberation Army 24: 29-31, 2012 (In Chinese).

16. Xu W, Jiang X, Xu Z, Ye T and Shi Q: The efficacy of Brucea javanica oil emulsion injection as adjunctive therapy for advanced non-small-cell lung cancer: A meta-analysis. Evid Based Complement Alternat Med 2016: 5928562, 2016.

(i) (5) This work is licensed under a Creative Commons Attribution-NonCommercial-NoDerivatives 4.0 International (CC BY-NC-ND 4.0) License. 